\title{
The effect of using cow genomic information on accuracy and bias of genomic breeding values in a simulated Holstein dairy cattle population
}

\author{
E. Dehnavi, ${ }^{\star} \dagger$ S. Ansari Mahyari, ${ }^{* 1}$ F. S. Schenkel, $†$ and M. Sargolzaei†‡ \\ *Department of Animal Science, College of Agriculture, Isfahan University of Technology, Isfahan, 84156-83111, Iran \\ †Centre for Genetic Improvement of Livestock, Department of Animal Biosciences, University of Guelph, Guelph, ON N1G 2W1, Canada \\ ‡Semex Alliance, Guelph, ON N1H 6J2, Canada
}

\begin{abstract}
Using cow data in the training population is attractive as a way to mitigate bias due to highly selected training bulls and to implement genomic selection for countries with no or limited proven bull data. However, one potential issue with cow data is a bias due to the preferential treatment. The objectives of this study were to (1) investigate the effect of including cow genotype and phenotype data into the training population on accuracy and bias of genomic predictions and (2) assess the effect of preferential treatment for different proportions of elite cows. First, a 4-pathway Holstein dairy cattle population was simulated for 2 traits with low (0.05) and moderate (0.3) heritability. Then different numbers of cows $(0,2,500,5,000,10,000,15,000$, or $20,000)$ were randomly selected and added to the training group composed of different numbers of top bulls $(0,2,500,5,000,10,000$, or 15,000$)$. Reliability levels of de-regressed estimated breeding values for training cows and bulls were 30 and $75 \%$ for traits with low heritability and were 60 and $90 \%$ for traits with moderate heritability, respectively. Preferential treatment was simulated by introducing upward bias equal to $35 \%$ of phenotypic variance to 5,10 , and $20 \%$ of elite bull dams in each scenario. Two different validation data sets were considered: (1) all animals in the last generation of both elite and commercial tiers $(\mathrm{n}=42,000)$ and $(2)$ only animals in the last generation of the elite tier $(\mathrm{n}=$ 12,000). Adding cow data into the training population led to an increase in accuracy $(r)$ and decrease in bias of genomic predictions in all considered scenarios without preferential treatment. The gain in $\mathrm{r}$ was higher for the low heritable trait (from 0.004 to $0.166 \mathrm{r}$ points) compared with the moderate heritable trait (from 0.004 to $0.116 \mathrm{r}$ points). The gain in accuracy in scenarios with a lower number of training bulls was relatively
\end{abstract}

Received April 8, 2017.

Accepted December 17, 2017.

${ }^{1}$ Corresponding author: s.ansari@cc.iut.ac.ir higher (from 0.093 to $0.166 \mathrm{r}$ points) than with a higher number of training bulls (from 0.004 to $0.09 \mathrm{r}$ points). In this study, as expected, the bull-only reference population resulted in higher accuracy compared with the cow-only reference population of the same size. However, the cow reference population might be an option for countries with a small-scale progeny testing scheme or for minor breeds in large counties, and for traits measured only on a small fraction of the population. The inclusion of preferential treatment to 5 to $20 \%$ of the elite cows led to an adverse effect on both accuracy and bias of predictions. When preferential treatment was present, random selection of cows did not reduce the effect of preferential treatment.

Key words: prediction accuracy, cow genomic data, preferential treatment, regression coefficient

\section{INTRODUCTION}

Genomic selection has become a common practice in dairy cattle breeding programs due to an ever-decreasing cost of high-throughput genotyping products (Schefers and Weigel, 2012). Selection decisions in dairy cattle breeding programs are now being made based on genomic estimated breeding values (GEBV), which usually are available early in life and are more accurate than traditional parent average (Goddard and Hayes, 2009). This has resulted in shortening the generation interval significantly and consequently increasing the annual genetic gain.

In some countries, genomic evaluation is mainly based on a bull training population because of the higher accuracy of bull proofs. One problem with a bull-only training population is selection bias due to intense selection on bulls (Patry and Ducrocq, 2011; Schaeffer, 2014). In addition, the size of the training population and accuracy of observations are the most important factors determining the accuracy of genomic predictions (Goddard, 2009). Other factors, such as the extent of linkage disequilibrium (LD) and genetic architecture of the trait, play an important role as well (Goddard and Hayes, 2009). For GEBV of young 
animals, Wiggans et al. (2010) reported average reliability of $76 \%$ for production traits based on a training population of 7,173 Holstein proven bulls. However, for a smaller training population, which consisted of 3,576 proven bulls, an average expected reliability of $69 \%$ and observed reliability of 62 to $66 \%$ for production traits were reported by VanRaden et al. (2009). Therefore, countries with small bull training populations may have to cope with a lower accuracy of genomic predictions. Sharing genotypes and phenotypes with other countries to increase the size of training population seems to be a useful solution (VanRaden and Sullivan, 2010; Weller et al., 2015, 2017). However, collaboration with other countries increases the risk of genotype-environment interaction. The genotype-environment interaction is mainly due to genes that are differently expressed in different environments, leading to different SNP effects in each environment. An alternative way to increase the size of a training population is to include cow information (genotype and phenotype; Wensch-Dorendorf et al., 2011; Pryce et al., 2012). However, cow information needs to be incorporated cautiously because there might be bias due to preferential treatment (PT) of elite cows. Instead, using a randomly selected group of females or adjusting for potential PT bias could be beneficial. Moreover, there is a lower genetic variation among the bulls, because of more intense selection on sires. Therefore, adding cow information may provide some additional variation that can increase accuracy and reduce bias and, hence, may allow for potential young candidates to be ranked better for selection.

Generally, adding cow information to the training population is challenging, as results appear to vary depending on the implementation strategy used (Gao et al., 2015). Moreover, few studies have examined the effect of PT on the accuracy and bias of genomic breeding values. The benefit of using cow information in the training population mainly depends on the degree of $\mathrm{PT}$, the size of the cow training population, the size of the bull training population, and relationships with candidate females and the heritability of the phenotype. Therefore, the objectives of present study were to (1) investigate the effect of adding cow information into a training population on the accuracy and bias of genomic predictions and (2) assess the effect of PT bias when cow information is added to the training population, using a simulated Holstein cattle population.

\section{MATERIALS AND METHODS}

\section{Genome Structure}

A genome consisting of 29 autosomes each with different lengths similar to the bovine chromosomes for a total length of 2,496 $\mathrm{cM}$ was simulated (Bohmanova et al., 2010). Genotypes were simulated for 50,000 biallelic markers and 750 multi-allelic QTL $(2,3$, or 4 alleles) with initial equal allele frequencies, and random location on the genome. The effects of QTL alleles were sampled from a gamma distribution with a shape parameter of 0.4. The mutation rate for both SNP and QTL was set at $1 \times 10^{-4}$ under the recurrent mutation model (Sargolzaei and Schenkel, 2009). Interference rate was set to 25\% (Weeks et al., 1994; Tapper et al., 2002; Sargolzaei and Schenkel, 2009). The ascertainment bias in the SNP panel was introduced by selecting segregating loci according to MAF distribution observed in North American bull data.

\section{Population Structure}

A Holstein dairy cattle population was simulated using QMSim software (Sargolzaei and Schenkel, 2009). Supplemental Figure S1 (https://doi.org/10.3168/jds .2017-12999) presents the simulation schematic. First, a historical population was generated to reach mutationdrift equilibrium. To mimic AI and progeny test breeding schemes and to establish long-range $\mathrm{LD}$, an $\mathrm{AI}$ population was simulated. This population mimicked a 4-pathway dairy cattle selection program with the use of AI technology. After establishing the proper level of LD, a most recent population, consisting of the elite (i.e., nucleus) and commercial groups, was simulated for 6 generations under the genomic selection scheme (GS population).

In the GS population, the selection of young animals was based on a predefined reliability of $70 \%$, which represents the squared accuracy of genomic selection in the United States (VanRaden et al., 2009; Wiggans et al., 2010). Because a proven bull EBV is based on a large number of daughters, it is more accurate than a cow EBV based on a limited number of daughters and her own record. Therefore, pseudo-phenotypes for bulls and dams were simulated by calculating different predefined accuracies based on de-regressed EBV in Holstein cattle in Canada. Data were provided by Canadian Dairy Network and consisted of 7,500 domestically proven bulls and 5,000 cows. The bulls were those used in the training set of the national Canadian genomic evaluation. To simulate pseudo-phenotypes with a predefined accuracy, a correlated vector to true breeding values (TBV) was simulated as follows:

$$
\mathbf{V}=\left(\begin{array}{ll}
v a r & \operatorname{cov} \\
\operatorname{cov} & v a r
\end{array}\right)
$$

$\operatorname{cholesky}(\mathbf{V})=\mathbf{C C}^{\prime}$

Journal of Dairy Science Vol. 101 No. 6, 2018 


$$
\begin{aligned}
& \mathbf{X}= \\
& \left(\begin{array}{cc}
T B V / \sqrt{v a r} & N R N D \\
\vdots & \vdots
\end{array}\right) \times \mathbf{C}^{\prime}=\left(\begin{array}{cc}
T B V & \text { PseudoPhenotype } \\
\vdots & \vdots
\end{array}\right)
\end{aligned}
$$

where $\mathbf{V}$ is a (co)variance matrix of $\mathrm{TBV}$, in which covariance is calculated according to accuracy (i.e., correlation) and NRND is a normal random deviate; $\mathbf{C}$ is a lower triangular matrix with positive diagonal; and $\mathbf{C}^{\prime}$ is a transpose of matrix $\mathbf{C}$. The final produced matrix $(\mathbf{X})$ consists of 2 columns: TBV and pseudophenotypes. The simulated pseudo-phenotypes would represent de-regressed EBV.

The simulated pseudo-phenotypes were then used in the training group and mixed model equations were weighted according to the corresponding reliabilities. For a trait with low heritability $\left(\mathrm{h}^{2}=0.05\right)$, reliabilities of 30 and $75 \%$ for dams and sires were used and for a trait with moderate heritability $\left(\mathrm{h}^{2}=0.3\right)$, reliabilities of 60 and $90 \%$ were used, respectively.

The 2 traits, representing production and functional traits, were independently simulated. A phenotypic variance of 100 was considered for both traits. For comparison of the different scenarios, genomic predictions were carried out with a training population consisting of animals from generation 5 of the GS population, whereas generation 6 was used as a validation group. Generation 5 of the GS population was used as the training population to mimic the likely real scenario where genomic selection is an ongoing process and females are not widely genotyped in the early implementation of genomic selection (see Supplemental Figure S1; https://doi.org/10.3168/jds.2017-12999).

Two alternative validation groups were used. The first validation group consisted of all 42,000 individuals in generation 6 . The second validation group included only the top 12,000 individuals born in the elite tier. All individuals in these 2 validation groups were descendants of the training animals. To evaluate the effect of including cow genomic/phenotypic data in the training population on bias and accuracy of genomic predictions, different numbers of cows (i.e., 0, 2,500, $5,000,10,000,15,000$, and 20,000) were added to each bull training group composed of either 0, 2,500, 5,000, 10,000 , or 15,000 bulls. The simulation was replicated 20 times.

\section{Preferential Treatment Simulation}

The PT was simulated on the selected training population. The mean PT was simulated to be equal to $35 \%$ of the phenotypic variance, which is consistent with the effect attributable to the management (Kuhn et al., 1994; Weigel et al., 1994). Preferential treatment usually causes an upward bias as elite cows are treated better than the rest of the herd (Kuhn et al., 1994; Weigel et al., 1994). Therefore, PT was simulated by adding normal random deviates with a mean equal to $35 \%$ of the phenotypic variance and a standard deviation (SD) equal to one-third of the mean. The minimum and maximum values for this distribution were set to mean $-3 \times \mathrm{SD}$ and mean $+3 \times \mathrm{SD}$, respectively. This restriction prevents negative $\mathrm{PT}$ values. Then this bias was added to 5,10 , and $20 \%$ of top dams in each cow training group consisted of either 2,500, 5,000, 10,000, 15,000 , or 20,000 cows. The top cows were identified based on their pseudo-phenotypes. To assess whether a random selection of cows could reduce the effect of $\mathrm{PT}$, another scenario was considered, where cows for the training population were randomly selected from a population of cows in which $10 \%$ were preferentially treated.

\section{Comparison Criteria}

Pearson correlation between TBV and predicted direct genomic value (DGV) and regression coefficient of TBV on DGV were used as measurement criteria of accuracy and bias, respectively. Expected regression coefficient $(E x b)$ was also calculated as

$$
E x b=\{1-[i(i-x)]\} /\left\{1-\left[i(i-x) \mathrm{R}^{2}\right]\right\}
$$

where $i$ and $x$ were the standardized selection differential and selection differential for genotyped individuals in the training population, respectively, and $R^{2}$ is the coefficient of determination with respect to regression coefficient of TBV on DGV for unselected validation individuals (Olson et al., 2011).

\section{Genomic Evaluation}

The genomic evaluation was carried out using the following model:

$$
y_{i}=\mu+\mathbf{W}_{i} u_{i}+\sum_{j=1}^{p} \beta_{j} X_{i j}+e_{i}
$$

where $y_{i}$ is the observation for individual $i, \mu$ is the overall mean, $u_{i}$ is the random polygenic effect of individual $i, \beta_{j}$ is the regression coefficient for $\operatorname{SNP} j, e_{i}$ 
is the residual, $\mathbf{W}_{i}$ is an incidence matrix that links records to individual $i$, and $X_{i j}$ is

$$
\left(\mathbf{Z}_{i j}-2 f_{j}\right) / \sqrt{2 \sum_{j=1}^{p}\left[f_{j}\left(1-f_{j}\right)\right]},
$$

where $f_{j}$ is frequency of allele 1 for $\operatorname{SNP} j$ and $\mathbf{Z}$ is a genotype matrix for $n$ animals and $p$ SNP.

Genomic predictions based on the above model were computed using the snpBLUP method in SNP1101 software (Sargolzaei, 2014).

\section{RESULTS AND DISCUSSION}

\section{LD Structure, Chromosome Structure, Ascertainment Bias}

The extent of LD in the simulated AI population showed a similar pattern but was slightly higher than that observed in North American Holstein cattle (Supplemental Figure S2; https://doi.org/10.3168/jds.2017 -12999). The recent and past effective population sizes $(\mathrm{Ne})$ are given in Supplemental Figure S3 (https://doi .org/10.3168/jds.2017-12999). The simulated recent Ne matched well with that observed in North American Holsteins (Bohmanova et al., 2010).

In this study, the number of chromosomes and their length were based on real data except for $\mathrm{X}$ and $\mathrm{Y}$ chromosomes, which were not considered. Accounting for different chromosome lengths in addition to the number of chromosomes is an important aspect of genome simulation. Longer chromosomes are more likely to experience more recombination events than shorter ones (Arias et al., 2009). Moreover, different chromosomes harbor different numbers of QTL.

As commercial SNP chips were designed by selecting SNP that segregate with a minimum rare allele frequency (Kuhner et al., 2000; Nielsen, 2000, 2004), an ascertainment bias was simulated by selecting segregating SNP according to the minor allele frequency (MAF) distribution observed in North American Holstein data (Supplemental Figure S4; https://doi.org/ 10.3168/jds.2017-12999). The average MAF before and after introducing ascertainment bias were 0.21 and 0.24 , respectively.

\section{Validation Results Using Nonselected Animals}

For this scenario, All 42,000 animals born in generation 6 were used in the validation group, without any selection.

Accuracy of $\boldsymbol{D G} \boldsymbol{V}$. The accuracy from cow-only training population was considerably lower compared with the corresponding proven bull-only scenario with the same training size (Figure 1). This was mainly because of the lower accuracy of cows' EBV. However, the use of the cow-only training population might be an alternative for countries that do not have enough number of proven bulls due to lack of progeny test schemes. Including cow evaluations is also an important option for minor breeds in large counties, and for traits measured only on a small fraction of the population. In this case, several thousands of cows should be genotyped and phenotyped for achieving a reasonable prediction accuracy (Figure 1).

Adding cow genotypic and phenotypic information to the training population resulted in an increase in the accuracy $(r)$ of genomic predictions in all scenarios when compared with the bull-only training population (Figures 2, panel 5, and Figure 3, panel 5). The gain in accuracy (r) for different scenarios ranged from 0.004 to 0.116 points for a moderate heritable trait $\left(h^{2}=0.30\right.$;

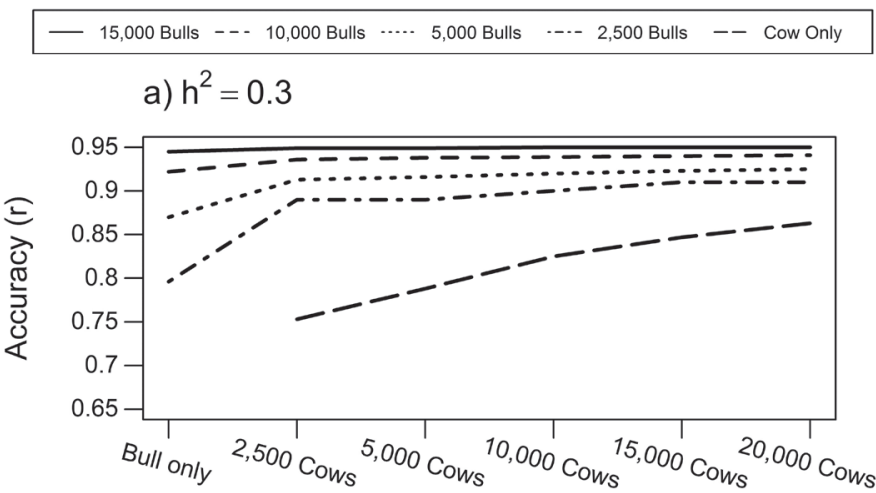

b) $h^{2}=0.05$

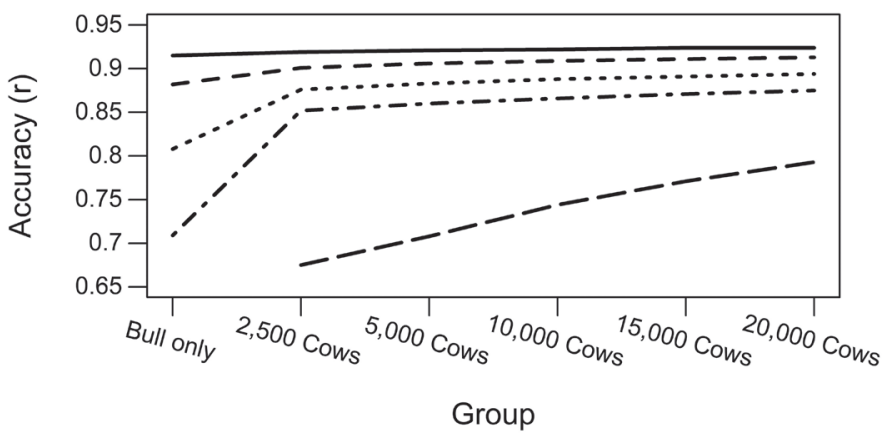

Figure 1. Accuracy (r) in validation populations for different training populations for 2 traits [a: $\mathrm{h}^{2}=0.3$ and $\left.\mathrm{b}: \mathrm{h}^{2}=0.05\right]$. Different training populations consisted of using only bulls in different numbers (i.e., 2,500, 5,000, 10,000, and 15,000) in the training population, using only cows in different numbers (i.e., 2,500, 5,000, 10,000, 15,000, and 20,000) in the training population, or combination of 2 sexes. Excluding the curve related to the cow-only training population, each curve starts with the accuracy of using bulls only in the training population at the first point and continues with results of accuracy after adding different numbers of cows in the next points. 
1) with $5 \% \mathrm{PT}$

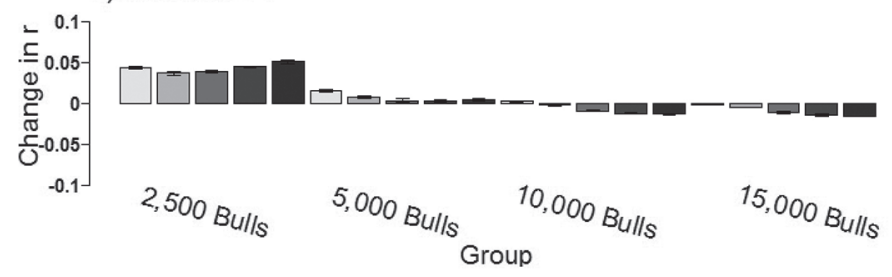

3) with $20 \% \mathrm{PT}$

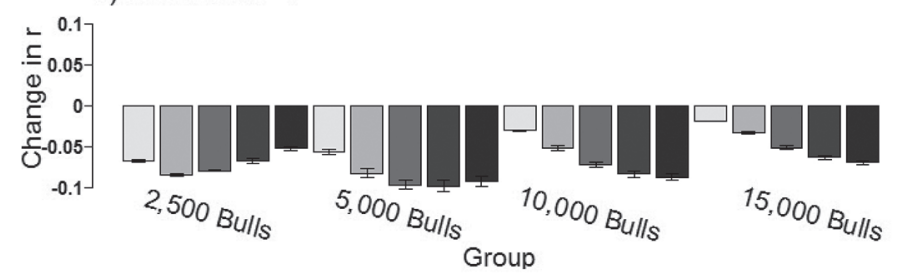

5) No PT

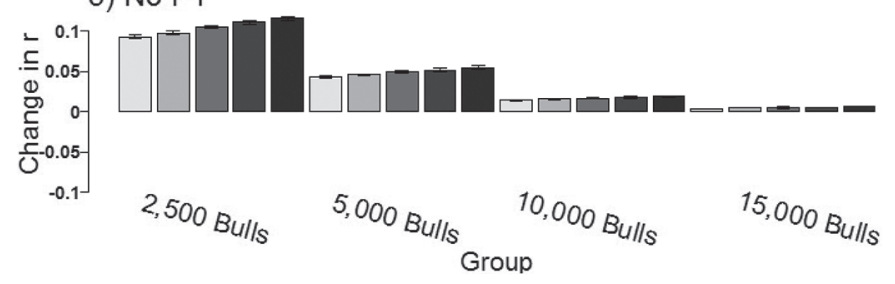

2) with $10 \%$ PT

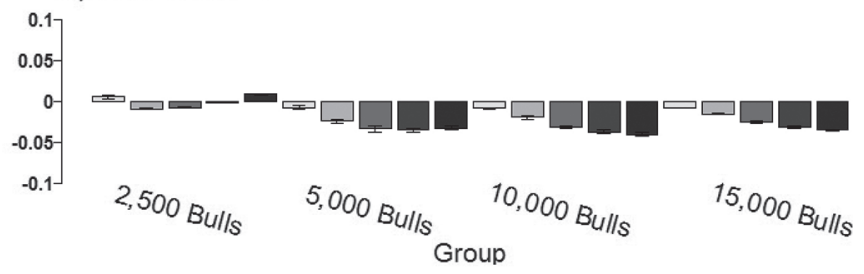

4) Random Selection

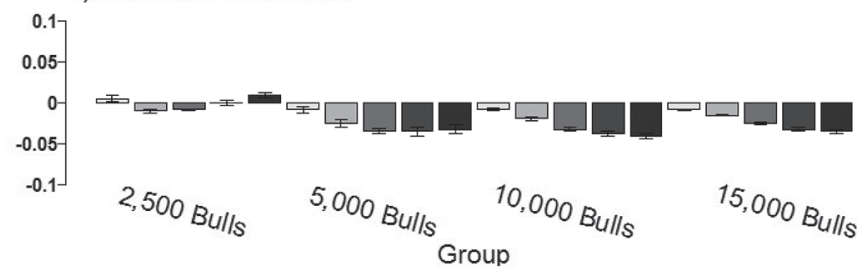

Figure 2. Change in accuracy $(\mathrm{r})$ including preferentially treated cows in the training population for a trait with $\mathrm{h}^{2}=0.30$. The change was the subtraction of accuracy of validation population by using different numbers of cows and bulls (i.e., 2,500, 5,000, 10,000, 15,000, and 20,000) in the training population from the accuracy of validation population by using the bull-only training population. A total of 5 , 10 , and $20 \%$ of top cows in each group of 2,500,5,000,10,000,15,000, and 20,000 cows received preferential treatment. Panel 4 shows differences in accuracy after random selection of cows from a training population that included preferential treatment (PT) for $10 \%$ of elite cows. Error bars represent SE for each scenario.

Figure 2, panel 5) and from 0.004 to 0.166 points for a low heritable trait $\left(h^{2}=0.05\right.$; Figure 3 , panel 5$)$. As expected, the gain was higher for the trait with lower heritability. The use of cow information resulted in a higher gain in scenarios with a lower number of bulls in the training population. Including cow information to the training population, which included only 2,500 proven sires, resulted in a gain in accuracy from 0.093 to 0.166 points for the 2 traits (Figures 2, panel 5, and Figure 3, panel 5). This higher gain than in scenarios with more proven sires in the training population was expected because a larger number of proven bulls in the training population alone provided enough information to explain much of genetic variation of the population.

The results of accuracy are in agreement with previous studies (Mc Hugh et al., 2011; Wiggans et al., 2011; Ding et al., 2013; Tsuruta et al., 2013; Gao et al., 2015). Using simulation, Mc Hugh et al. (2011) reported an accuracy of GEBV between 0.74 and 0.83 for young males, and 0.67 and 0.85 for the young female population. Tsuruta et al. (2013), using a US Holstein population, assessed change in reliability and bias when information of 5,235 cows was added to training group of 34,506 bulls in the multi-step genomic evaluation.
The reliabilities were increased by 1.3 to $3.9 \%$, and no significant improvement was present in the bias when compared with the use of only bulls in the training population. Including 10,000 cows' genomic information into an Australian Holstein training population of 3,000 bulls could lead to an improvement of $8 \%$ in the reliabilities (Pryce et al., 2012). Therefore, for situations where the size of the proven bull training group is small, incorporating female data to the training population could be beneficial (e.g., in Fleckvieh, Edel et al., 2016; in beef cattle, Gunia et al., 2014; and in chickens, Lourenco et al., 2015). Therefore, one way to increase the reliability of genomic prediction and, hence, to increase the genetic gain in a small population, is to use cow information in the training data set (Thomasen et al., 2014). Ding et al. (2013) used a training population of 3,087 Chinese Holstein cows and reported a 0.30 to 0.33 point gain in accuracy over traditional EBV (Ding et al., 2013). For Jersey breed, Gao et al. (2015) found 1.9 to 4.5 percentage points improvement in the reliability of genomic predictions by including genotyped and phenotyped females besides proven bulls in the training population. However, in some breeds adding cow information did not increase 

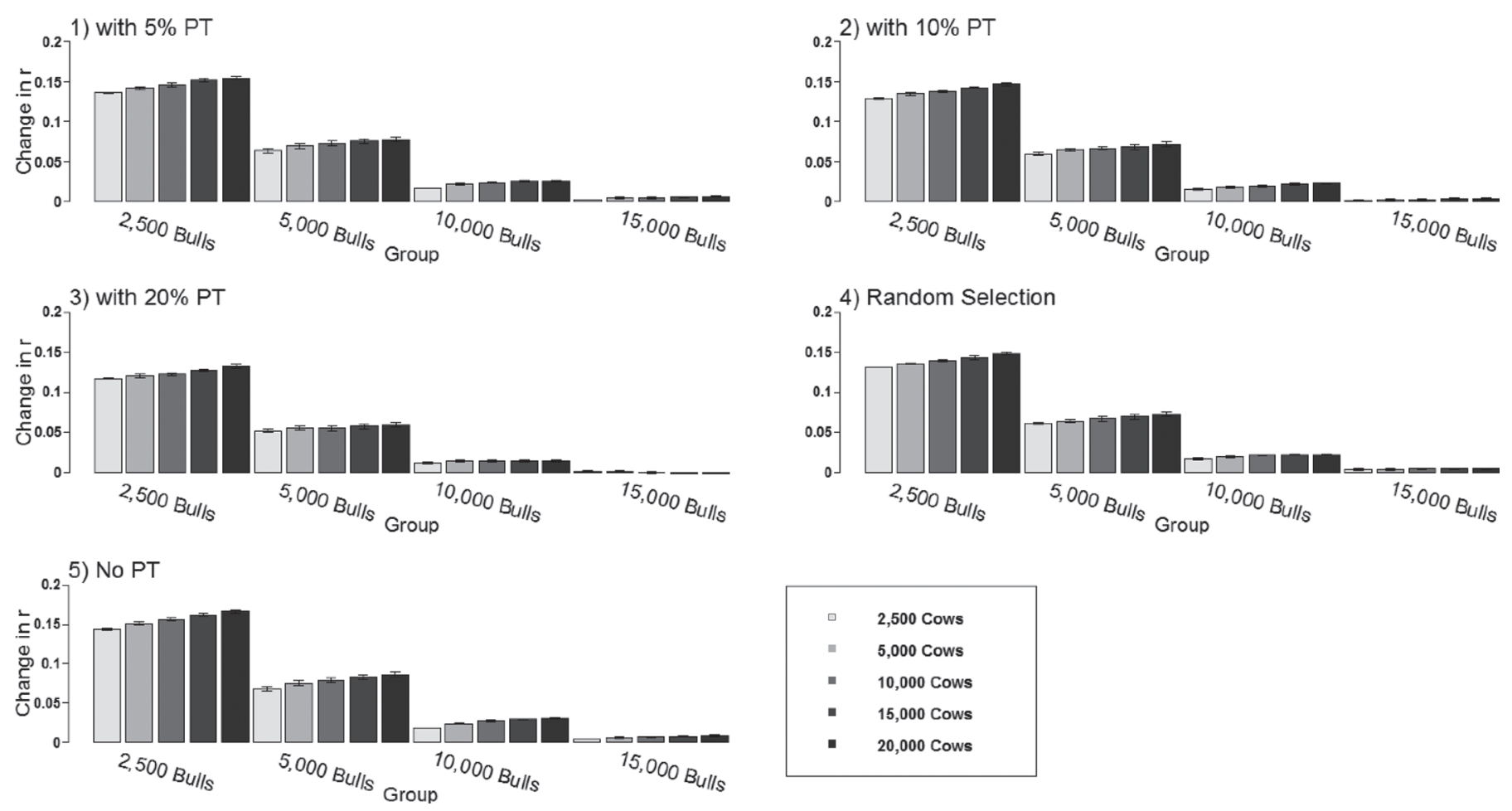

Figure 3. Change in accuracy (r) including preferentially treated cows in the training population for a trait with $\mathrm{h}^{2}=0.05$. The change was the subtraction of accuracy of validation population by using different numbers of cows and bulls (i.e., 2,500, 5,000, 10,000, 15,000, and 20,000) in the training population from the accuracy of validation population by using the bull-only training population. A total of 5 , 10 , and $20 \%$ of top cows in each group of $2,500,5,000,10,000,15,000$, and 20,000 cows received preferential treatment. Panel 4 shows differences in accuracy after random selection of cows from a training population that included preferential treatment (PT) for $10 \%$ of elite cows. Error bars represent SE for each scenario.

the accuracy of genomic evaluations significantly, such as those reported for Brown Swiss population (Bapst et al., 2013). This may relate to the number of cows included in the training population, the reliability of observations, the trait, or SNP density. Wiggans et al. (2011) improved the reliability of genomic evaluations by adding adjusted cow PTA to the training population, which included only bulls. They adjusted cows for possible inflation of PTA, which may originate from PT. Average gains of 0.53 and 0.57 percentage points in reliability were obtained for Holsteins and Jerseys, respectively, using adjusted cows plus bulls in the training population compared with using only bulls in the training population (Wiggans et al., 2011).

In this study, the bull-only reference population resulted in higher accuracy compared with the cow-only reference population of the same size (Figure 1). This was expected because cows had lower reliability compared with proven sires. This was in agreement with previous studies (Wensch-Dorendorf et al., 2011; Gunia et al., 2014; Thomasen et al., 2014; Chesnais et al., 2016) and indicated that a larger number of cows is required to reach the same level of accuracy as obtained using proven bulls.
In this study, the parents of validation animals were in the training data set. Therefore, close relationships between validation and training individuals might have contributed to the observed high correlation between DGV and TBV. The accuracy of genomic prediction increases by minimizing the relatedness between individuals within the training population and maximizing the relatedness between individuals in the training population and the validation population (Bapst et al., 2013). In the current study, the average additive relationship between animals in the training population was 0.08 . Moreover, the average additive relationship between animals in the training and validation populations was similar on the sire side compared with the dam side (0.09 and 0.08, respectively).

Regression Coefficient and Expected Regression Coefficient. The coefficient of regression (b) of TBV on DGV improved and became closer to 1 when cow information with no PT was incorporated into the training group compared with the bull-only training population in all scenarios (Table 1). This was expected because there was no $\mathrm{PT}$ in the first simulated data set. Results in Table 1 also show that intense selection on training bulls led to more bias. As there was no 
intense selection in the cows' training group, less bias was observed. However, it should be noted that training cows were also affected by the more intense selection on training bulls through their sires, but to a lesser degree. Therefore, adding the information of cows to the training population could be helpful to reduce bias. Patry and Ducrocq (2011) also argued that pre-selection of young animals based on GEBV (i.e., selective phenotyping) leads to an underestimation of their BLUP $\mathrm{EBV}$, and consequently this downward bias is spread to the rest of population through SNP effects (e.g., validation animals). In the present simulation study, DGV of validation animals were underestimated for all scenarios (Table 1), whereas in other studies that were carried out on real data, DGV were mostly overestimated (e.g., Wiggans et al., 2011; Tsuruta et al., 2013; Gunia et al., 2014; Gao et al., 2015). This is due to the fact that in real dairy data proven animals are usually chosen for validation, which represents a highly selected sample of proven bulls. In this simulation study, all individuals born in the last simulated generation were included in the validation group, regardless of their genetic merit, because they had TBV to compare with their DGV. When an alternate validation group, consisting of selected animals in the elite tier, was used, the DGV were overestimated as in the previously mentioned studies with real dairy data sets (see the Validation Results Using Top Selected Animals section for details).

Preferential Treatment. Figures 2 and 3 depict the effect of using cows in the training population on the prediction accuracy when there was PT. The moderate heritable trait $\left(h^{2}=0.30\right.$, Figure 2) was more affected by PT than the lower heritability trait $\left(\mathrm{h}^{2}=\right.$ 0.05 , Figure 3). Increasing the number of individuals in the training population resulted in a reduction in the effect of PT bias (Figures 2 and 3). Increasing the number of individuals in the training population resulted in the increase of nonbiased information and, hence, reduction of deteriorating effect of PT. Table 2 shows that random selection of cows is not an effective strategy to reduce the bias due to PT. Nevertheless, Dassonneville et al. (2012) reported lower correlations between GEBV predicted when the training population included both bulls' daughter yield deviations (DYD) and cows' own yield deviations and GEBV predicted when the training population included only bull daughter yield deviations for elite cows than for randomly selected cows for milk yield.

Tables 3 and 4 present coefficients of regression of TBV on DGV for a scenario where 5 to $20 \%$ of top cows received PT. Because PT bias is usually an upward bias, it led to a reduction in regression coefficients, which became closer to 1 (Tables 3 and 4). Dassonneville et al. (2012) also demonstrated that including own milk

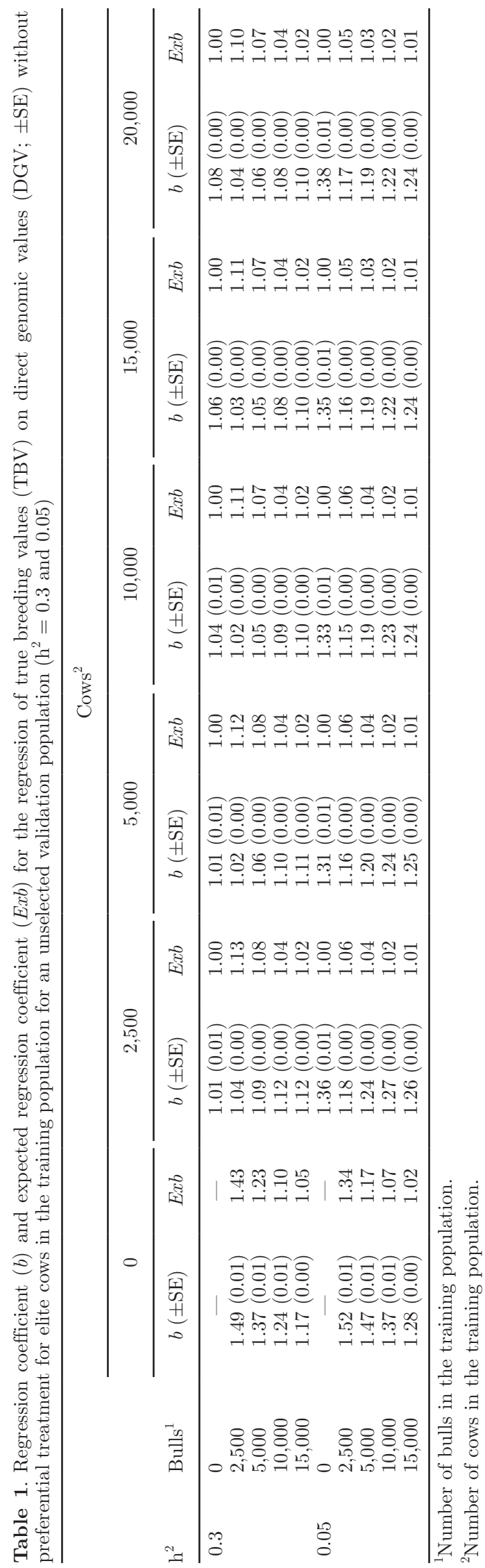


Table 2. Regression coefficient $(b)$ for the regression of true breeding values on direct genomic values $( \pm \mathrm{SE})$ in the unselected validation population after random selection of cows from a training population with preferential treatment on $10 \%$ of elite cows $\left(\mathrm{h}^{2}=0.3\right.$ and 0.05$)$

\begin{tabular}{|c|c|c|c|c|c|c|c|}
\hline$h^{2}$ & Bulls $^{1}$ & \multicolumn{6}{|c|}{ Cows $^{2}$} \\
\hline \multirow{4}{*}{0.3} & 2,500 & $1.49(0.01)$ & $0.86(0.01)$ & $0.79(0.01)$ & $0.75(0.01)$ & $0.74(0.01)$ & $0.74(0.01)$ \\
\hline & 5,000 & $1.37(0.01)$ & $0.99(0.00)$ & $0.91(0.01)$ & $0.85(0.01)$ & $0.83(0.01)$ & $0.82(0.00)$ \\
\hline & 10,000 & $1.24(0.01)$ & $1.08(0.00)$ & $1.02(0.00)$ & $0.96(0.00)$ & $0.93(0.00)$ & $0.91(0.00)$ \\
\hline & 15,000 & $1.17(0.00)$ & $1.1(0.00)$ & $1.06(0.00)$ & $1.01(0.00)$ & $0.98(0.00)$ & $0.96(0.00)$ \\
\hline \multirow{3}{*}{0.05} & 5,000 & $1.47(0.01)$ & $1.23(0.00)$ & $1.19(0.00)$ & $1.17(0.00)$ & $1.16(0.00)$ & $1.16(0.00)$ \\
\hline & 10,000 & $1.37(0.01)$ & $1.27(0.00)$ & $1.24(0.00)$ & $1.22(0.00)$ & $1.21(0.00)$ & $1.21(0.00)$ \\
\hline & 15,000 & $1.28(0.00)$ & $1.26(0.00)$ & $1.24(0.00)$ & $1.23(0.00)$ & $1.23(0.00)$ & $1.23(0.00)$ \\
\hline
\end{tabular}

${ }^{1}$ Number of bulls in the training population.

${ }^{2}$ Number of cows in the training population.

performance of elite females leads to overestimation genomic evaluations. Some studies support the idea that PT occurs among elite cows (Dassonneville et al., 2012; Murphy et al., 1982), whereas another study did not report any evidence of PT (Graham et al., 1991). When the aim is to use cows in the training population, potential bull dams are the first targeted females. The use of potentially biased records of elite cows in genomic evaluation may have an effect on GEBV of the cows themselves and their relatives. In this study, PT was limited to the elite cows themselves and was not propagated to their relatives' EBV. Therefore, results here are a conservative assessment of the potential bias due to PT in genomic selection. Since the implementation of genomic selection in dairy cattle, only a few studies have addressed the problem of PT (Wiggans et al., 2011; Calus et al., 2016). Given that PT is not recorded or poorly recorded, adjusting for $\mathrm{PT}$ is not trivial. Therefore, further research on proper adjustment for PT is needed using genomic information.

\section{Validation Results Using Top Selected Animals}

According to Table 5, when validation animals were selected based on high EBV, their DGV were overestimated, similarly to results found in studies on real data (e.g., Wiggans et al., 2011; Tsuruta et al., 2013; Gunia et al., 2014; Gao et al., 2015). When applying genomic selection in practice, selection candidates (validation animals here) are often not a random sample of candidates, but rather individuals from a highly selected group of animals. After selection, the variance of the

Table 3. Regression coefficient $(b)$ of the regression of true breeding values on direct genomic values $( \pm \mathrm{SE})$ with preferential treatment $(\mathrm{PT})$ for 5,10 , and $20 \%$ of elite cows in the training population for an unselected validation population $\left(\mathrm{h}^{2}=0.3\right)$

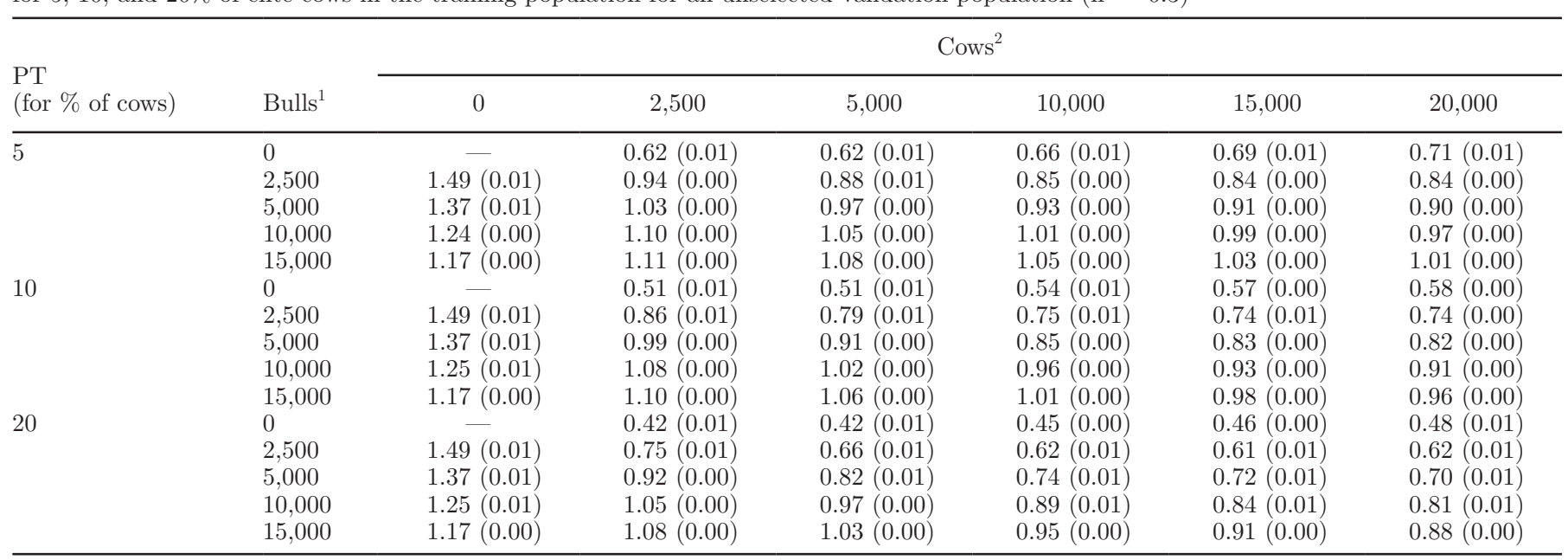

${ }^{1}$ Number of bulls in the training population.

${ }^{2}$ Number of cows in the training population. 
Table 4. Regression coefficient $(b)$ of the regression of true breeding values on direct genomic values $( \pm \mathrm{SE})$ with preferential treatment $(\mathrm{PT})$ for 5,10 , and $20 \%$ of elite cows in the training population for an unselected validation population $\left(\mathrm{h}^{2}=0.05\right)$

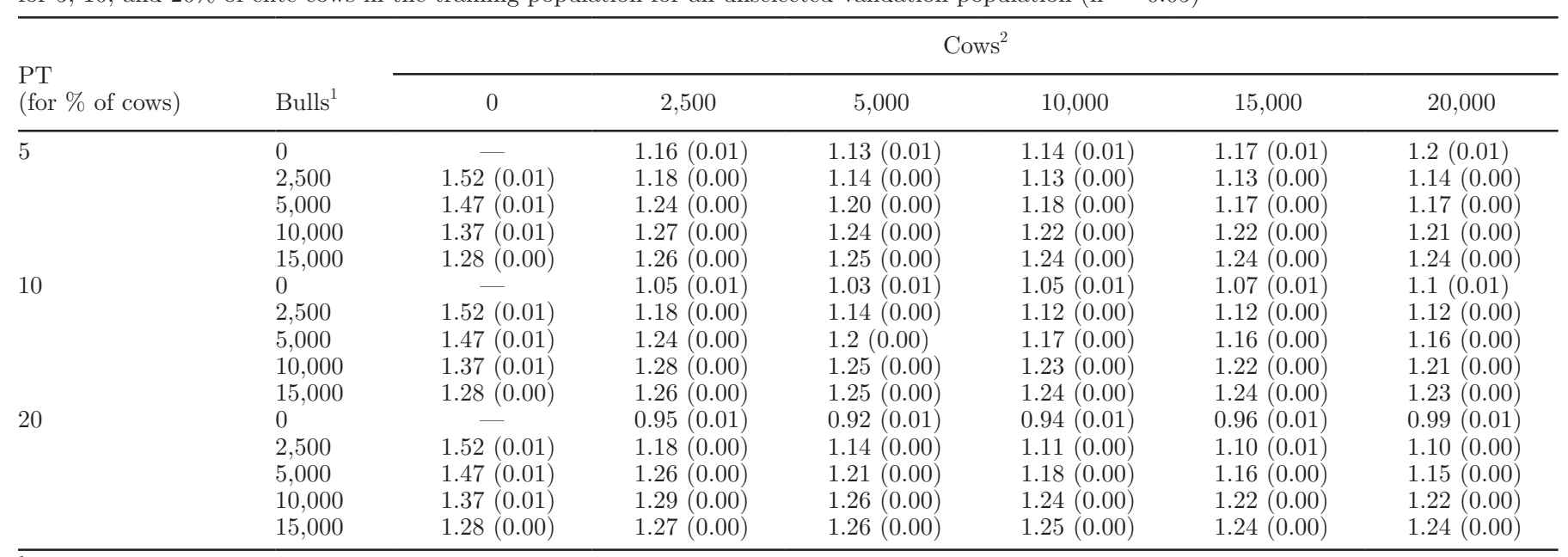

${ }^{1}$ Number of bulls in the training population.

${ }^{2}$ Number of cows in the training population.

selected candidates is reduced and their mean EBV tends to be higher than for the entire population of bulls. An underestimation of the genetic variance leads to overestimation of the breeding values (Weigel and Banos, 1997). Schaeffer (2014) pointed out that EBV of pre-selected individuals can be overestimated and hence their GEBV would potentially be overestimated as well. Results of the current study in terms of coefficient of regression of TBV on DGV with a selected validation population are in line with his point.

\section{CONCLUSIONS}

The inclusion of cow information in the training population resulted in a slight increase in accuracy of genomic prediction and a slight reduction in bias com- pared with the use of only proven bulls in the training population in all investigated scenarios. When the number of training bulls was relatively small (i.e., 2,500), adding cow information to the training group led to a higher gain in accuracy of DGV compared with the situation when the number of training bulls was large (i.e., 15,000). A cow-only training population might be a suitable option for countries with no progeny testing schemes. However, a larger cow training population is needed in this case. Preferential treatment of top cows deteriorated both prediction accuracy and bias. Random selection of cows, which might include preferentially treated cows, did not reduce the bias. The DGV of validation animals were underestimated in all scenarios when the validation animals were not selected but were overestimated when the validation set included only elite candidates.

Table 5. Regression coefficient $(b)$ of true breeding values on direct genomic values $( \pm \mathrm{SE})$ without preferential treatment for elite cows in the training population for a selected validation population for 2 traits $\left(h^{2}=0.3\right.$ and 0.05$)$

\begin{tabular}{|c|c|c|c|c|c|c|c|}
\hline$h^{2}$ & Bulls $^{1}$ & \multicolumn{6}{|c|}{ Cows $^{2}$} \\
\hline \multirow[t]{4}{*}{0.3} & 0 & - & $0.43(0.01)$ & $0.48(0.01)$ & $0.55(0.01)$ & $0.61(0.01)$ & $0.65(0.01)$ \\
\hline & 5,000 & $0.90(0.01)$ & $0.81(0.01)$ & $0.80(0.01)$ & $0.80(0.01)$ & $0.80(0.01)$ & $0.81(0.01)$ \\
\hline & 10,000 & $0.94(0.01)$ & $0.90(0.01)$ & $0.89(0.01)$ & $0.88(0.01)$ & $0.88(0.01)$ & $0.88(0.01)$ \\
\hline & 15,000 & $0.95(0.01)$ & $0.94(0.01)$ & $0.93(0.01)$ & $0.93(0.01)$ & $0.92(0.01)$ & $0.93(0.01)$ \\
\hline \multirow{3}{*}{0.05} & 5,000 & $0.84(0.01)$ & $0.82(0.01)$ & $0.82(0.01)$ & $0.81(0.01)$ & $0.82(0.01)$ & $0.83(0.01)$ \\
\hline & 10,000 & $0.92(0.01)$ & $0.9(0.01)$ & $0.9(0.01)$ & $0.9(0.01)$ & $0.9(0.01)$ & $0.91(0.01)$ \\
\hline & 15,000 & $0.95(0.01)$ & $0.95(0.01)$ & $0.95(0.01)$ & $0.95(0.01)$ & $0.96(0.01)$ & $0.96(0.01)$ \\
\hline
\end{tabular}

${ }^{1}$ Number of bulls in the training population.

${ }^{2}$ Number of cows in the training population 


\section{ACKNOWLEDGMENTS}

This study was funded by Iran's Ministry of Science, Research and Technology (Tehran, Islamic Republic of Iran), by a contribution from the Dairy Research Cluster Initiative (Dairy Farmers of Canada, Ottawa; Agriculture and Agri-Food Canada, Guelph; the Canadian Dairy Network, Guelph; and the Canadian Dairy Commission, Ottawa), and partially by The Semex Alliance (Guelph, Canada). The Canadian Dairy Network (Guelph) provided the Holstein genotypes used in the linkage disequilibrium analysis.

\section{REFERENCES}

Arias, J. A., M. Keehan, P. Fisher, W. Coppieters, and R. Spelman. 2009. A high density linkage map of the bovine genome. BMC Genet. 10:18. https://doi.org/10.1186/1471-2156-10-18.

Bapst, B., C. Baes, F. R. Seefried, A. Bieber, H. Simianer, and B. Gredler. 2013. Effect of cows in the reference population: First results in Swiss Brown Swiss. Pages 187-191 in Proc. Interbull Bull. Nantes, France, Interbull, Uppsala, Sweden.

Bohmanova, J., M. Sargolzaei, and F. S. Schenkel. 2010. Characteristics of linkage disequilibrium in North American Holsteins. BMC Genomics 11:421. https://doi.org/10.1186/1471-2164-11-421.

Calus, M. P., J. Vandenplas, J. Ten Napel, and R. F. Veerkamp. 2016. Validation of simultaneous de-regression of cow and bull breeding values and derivation of appropriate weights. J. Dairy Sci. 99:6403-6419. https://doi.org/10.3168/jds.2016-11028.

Chesnais, J. P., T. A. Cooper, G. R. Wiggans, M. Sargolzaei, J. E. Pryce, and F. Miglior. 2016. Using genomics to enhance the selection of novel traits in North American dairy cattle. J. Dairy Sci. 99:2413-2427. https://doi.org/10.3168/jds.2015-9970.

Dassonneville, R., A. Baur, S. Fritz, D. Boichard, and V. Ducrocq. 2012. Inclusion of cow records in genomic evaluations and impact on bias due to preferential treatment. Genet. Sel. Evol. 44:40. https://doi.org/10.1186/1297-9686-44-40.

Ding, X., Z. Zhang, X. Li, S. Wang, X. Wu, D. Sun, Y. Yu, J. Liu, Y. Wang, Y. Zhang, S. Zhang, Y. Zhang, and Q. Zhang. 2013. The accuracy of genomic prediction for milk production traits in the Chinese Holstein population using a reference population consisting of cows. J. Dairy Sci. 96:5315-5323. https://doi.org/10.3168/ jds.2012-6194.

Edel, C., E. C. Pimentel, L. Plieschke, R. Emmerling, and K. U. Gotz. 2016. Short communication: The effect of genotyping cows to improve the reliability of genomic predictions for selection candidates. J. Dairy Sci. 99:1999-2004. https://doi.org/10.3168/jds .2015-10246.

Gao, H., P. Madsen, U. S. Nielsen, G. P. Aamand, G. Su, K. Byskov, and J. Jensen. 2015. Including different groups of genotyped females for genomic prediction in a Nordic Jersey population. J. Dairy Sci. 98:9051-9059. https://doi.org/10.3168/jds.2015-9947.

Goddard, M. E. 2009. Genomic selection: Prediction of accuracy and maximization of long-term response. Genetica 136:245-257. https://doi.org/10.1007/s10709-008-9308-0.

Goddard, M. E., and B. J. Hayes. 2009. Mapping genes for complex traits in domestic animals and their use in breeding programs. Nat. Rev. Genet. 10:381-391. https://doi.org/10.1038/nrg2575.

Graham, N. J., C. Smith, and J. P. Gibson. 1991. Investigation of preferential treatment for milk yield in Canadian Holsteins. Can. J. Anim. Sci. 71:21-27.

Gunia, M., R. Saintilan, E. Venot, C. Hoze, M. N. Fouilloux, and F. Phocas. 2014. Genomic prediction in French Charolais beef cattle using high-density single nucleotide polymorphism markers. J. Anim. Sci. 92:3258-3269. https://doi.org/10.2527/jas.2013-7478.
Kuhn, M. T., P. J. Boettcher, and A. E. Freeman. 1994. Potential biases in predicted transmitting abilities of females from preferential treatment. J. Dairy Sci. 77:2428-2437. https://doi.org/10.3168/jds .S0022-0302(94)77185-X.

Kuhner, M. K., P. Beerli, J. Yamato, and J. Felsenstein. 2000. Usefulness of single nucleotide polymorphism data for estimating population parameters. Genetics 156:439-447.

Lourenco, D. A. L., B. O. Fragomeni, S. Tsuruta, I. Aguilar, B. Zumbach, R. J. Hawken, A. Legarra, and I. Misztal. 2015. Accuracy of estimated breeding values with genomic information on males, females, or both: an example on broiler chicken. Genet. Sel. Evol. 47:56. https://doi.org/10.1186/s12711-015-0137-1.

Mc Hugh, N., T. H. Meuwissen, A. R. Cromie, and A. K. Sonesson. 2011. Use of female information in dairy cattle genomic breeding programs. J. Dairy Sci. 94:4109-4118. https://doi.org/10.3168/jds .2010-4016.

Murphy, P. A., R. W. Everett, and L. D. VanVleck. 1982. Comparison of first lactations and all lactations of dams to predict sons' milk evaluations. J. Dairy Sci. 65:1999-2005. https://doi.org/10.3168/ jds.S0022-0302(82)82450-8.

Nielsen, R. 2000. Estimation of population parameters and recombination rates from single nucleotide polymorphisms. Genetics 154:931-942.

Nielsen, R. 2004. Population genetic analysis of ascertained SNP data. Hum. Genomics 1:218-224. https://doi.org/10.1186/1479-7364-1-3 -218 .

Olson, K. M., P. M. VanRaden, M. E. Tooker, and T. A. Cooper. 2011. Differences among methods to validate genomic evaluations for dairy cattle. J. Dairy Sci. 94:2613-2620. https://doi.org/10.3168/ jds.2010-3877.

Patry, C., and V. Ducrocq. 2011. Evidence of biases in genetic evaluations due to genomic preselection in dairy cattle. J. Dairy Sci. 94:1011-1020. https://doi.org/10.3168/jds.2010-3804.

Pryce, J. E., B. J. Hayes, and M. E. Goddard. 2012. Genotyping dairy females can improve the reliability of genomic selection for young bulls and heifers and provide farmers with new management tools. Proc 38th ICAR Conf, Cork, Ireland.

Sargolzaei, M. 2014. SNP1101 User's Guide. Version 1.0. HiggsGene Solutions Inc., Guelph, Ontario, Canada.

Sargolzaei, M., and F. Schenkel. 2009. QMSim: a large-scale genome simulator for livestock. Bioinformatics 25: https://doi.org/10 .1093/bioinformatics/btp045.

Schaeffer, L. R. 2014. Is The Animal Model Obsolete? Center for Genetic Improvement of livestock (CGIL), Department of Animal Bioscience, University of Guelph, Guelph, Ontario, Canada, Sep 19 - Dec 3. 2014. http://animalbiosciences.uoguelph.ca/sites/ default/files/wrkshop_0.pdf.

Schefers, J. M., and K. A. Weigel. 2012. Genomic selection in dairy cattle: Integration of DNA testing into breeding programs. Anim. Front. 2:4-9. https://doi.org/10.2527/af.2011-0032.

Tapper, W. J., X. Ke, N. E. Morton, and A. Collins. 2002. Recombination, interference and sequence: Comparison of chromosomes 21 and 22. Ann. Hum. Genet. 66:75-86. https://doi.org/10.1017/ S0003480001008946.

Thomasen, J. R., A. C. Sørensen, M. S. Lund, and B. Guldbrandtsen. 2014. Adding cows to the reference population makes a small dairy population competitive. J. Dairy Sci. 97:5822-5832. https://doi .org/10.3168/jds.2014-7906.

Tsuruta, S., I. Misztal, and T. J. Lawlor. 2013. Short communication: Genomic evaluations of final score for US Holsteins benefit from the inclusion of genotypes on cows. J. Dairy Sci. 96:3332-3335. https://doi.org/10.3168/jds.2012-6272.

VanRaden, P. M., and P. Sullivan. 2010. International genomic evaluation methods for dairy cattle. Genet. Sel. Evol. 42: https://doi .org/10.1186/1297-9686-42-7.

VanRaden, P. M., C. Van Tassell, G. Wiggans, T. Sonstegard, R. Schnabel, J. Taylor, and F. Schenkel. 2009. Invited review: Reliability of genomic predictions for North American Holstein bulls. J. Dairy Sci. 92: https://doi.org/10.3168/jds.2008-1514. 
Weeks, D. E., J. Ott, and G. M. Lathrop. 1994. Detection of genetic interference: Simulation studies and mouse data. Genetics $136: 1217-1226$.

Weigel, D. J., R. E. Pearson, and I. Hoeschele. 1994. Impact of different strategies and amounts of preferential treatment on various methods of bull-dam selection. J. Dairy Sci. 77:3163-3173. https://doi.org/10.3168/jds.S0022-0302(94)77259-3.

Weigel, K. A., and G. Banos. 1997. Effect of time period of data used in international dairy sire evaluations. J. Dairy Sci. 80:3425-3430. https://doi.org/10.3168/jds.S0022-0302(97)76318-5.

Weller, J. I., E. Ezra, and M. Ron. 2017. Invited review: A perspective on the future of genomic selection in dairy cattle. J. Dairy Sci. 100: https://doi.org/10.3168/jds.2017-12879.

Weller, J. I., W. M. Stoop, H. Eding, C. Schrooten, and E. Ezra. 2015. Genomic evaluation of a relatively small dairy cattle population by combination with a larger population. J. Dairy Sci. 98:4945-4955. https://doi.org/10.3168/jds.2014-9086.
Wensch-Dorendorf, M., T. Yin, H. H. Swalve, and S. Konig. 2011 Optimal strategies for the use of genomic selection in dairy cattle breeding programs. J. Dairy Sci. 94:4140-4151. https://doi.org/10 .3168/jds.2010-4101.

Wiggans, G. R., T. A. Cooper, P. M. Vanraden, and J. B. Cole. 2011. Technical note: Adjustment of traditional cow evaluations to improve accuracy of genomic predictions. J. Dairy Sci. 94:6188-6193. https://doi.org/10.3168/jds.2011-4481.

Wiggans, G. R., T. A. Cooper, P. M. VanRaden, and M. V. Silva. 2010. Increased reliability of genetic evaluations for dairy cattle in the United States from use of genomic information. Page 476 in Proc. 9th World Congr. Genet. Appl. Livest. Prod., Leipzig, Germany, German Society for Animal Science, Leipzig, Germany. (Abstr.) 